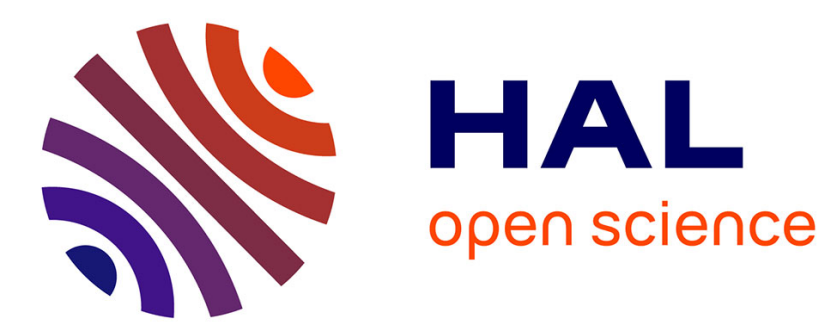

\title{
Strong generative capacity of RST, SDRT and discourse dependency DAGSs
}

\author{
Laurence Danlos
}

\section{To cite this version:}

Laurence Danlos. Strong generative capacity of RST, SDRT and discourse dependency DAGSs. A. Benz and P. Kuhnlein. Constraints in Discourse, Benjamins, pp.69-95, 2008. inria-00524739

\section{HAL Id: inria-00524739 \\ https://hal.inria.fr/inria-00524739}

Submitted on 8 Oct 2010

HAL is a multi-disciplinary open access archive for the deposit and dissemination of scientific research documents, whether they are published or not. The documents may come from teaching and research institutions in France or abroad, or from public or private research centers.
L'archive ouverte pluridisciplinaire HAL, est destinée au dépôt et à la diffusion de documents scientifiques de niveau recherche, publiés ou non, émanant des établissements d'enseignement et de recherche français ou étrangers, des laboratoires publics ou privés. 


\title{
Strong generative capacity of RST, SDRT and discourse dependency DAGSs
}

\author{
Laurence Danlos \\ LATTICE \\ Université Paris 7 \\ Institut Universitaire de France \\ laurence.danlos@linguist.jussieu.fr
}

\begin{abstract}
The aim of this paper ${ }^{0}$ is to compare the discourse structures proposed in RST, SDRT and dependency DAGs which extend the semantic level of MTT for discourses. The key question is the following: do these formalisms allow the representation of all the discourse structures which correspond to felicitous discourses and exclude those which correspond to infelicitous discourses? Hence the term of "strong generative capacity" taken from formal grammars.
\end{abstract}

\section{Introduction}

RST [Mann and Thompson1988] and SDRT [Asher and Lascarides2003] are quite different discourse theories, however they both rely on discourse relations and postulate an asymmetry: some parts of a text play a "subordinate" ("less important") role relative to other parts. This asymmetry is expressed in RST as a distinction between the arguments of discourse relations: arguments of type Nucleus are more important than arguments of type Satellite. This is expressed in SDRT as a distinction between the types of discourse relations: a coordinating relation links arguments of equal importance, while a subordinating relation links an important argument to a less important one. Since these distinctions come from the same idea, we use the following terminology for the two theories: a multi-nuclear or coordinating relation links two Nuclei ${ }^{1}$, while a nucleus-satellite or subordinating relations links a Nucleus and a Satellite.

On the other hand, dependency DAGs for discourse [Danlos2004], which extend the sentential semantic level of MTT (Meaning-Text Theory) [Mel'cuk1988] to the discourse level, relies on discourse relations but not on the distinction Nucleus/Satellite or coordinating/subordinating. This formalism only calls upon constraints coming from the semantic behavior of discourse connectives. These constraints are extrapolated to

\footnotetext{
${ }^{0}$ A French version of this paper is published in Revue TAL, Volume 47 Numéro 1, pp 169-198, 2006.

${ }^{1}$ A coordinating relation may link more than two Nuclei, e.g. Narration or Sequence. However, I leave aside this case, which means that it is supposed that all discourse relations discussed in this paper have exactly two arguments.
} 
discourse relations which are not lexicalized by a discourse connective. These (minimal) constraints are also respected in RST and SDRT (Section 4).

The aim of this paper is to compare RST, SDRT and discourse dependency DAGs with respect to the following question: do these formalisms allow the representation of all the discourse structures which correspond to felicitous discourses and exclude those which correspond to infelicitous discourses? By taking the term "strong generative capacity" from formal grammars, I reformulate this question as: what is the strong generative capacity of each of these three formalisms?

It will be shown that none of these formalisms has the appropriate strong generative capacity. RST, which imposes that discourse structures be tree shaped (among other constraints), is too restrictive (there exist some felicitous discourses whose structure is not controversial but not representable as an RST tree). At the opposite extreme, dependency DAG formalism, which only imposes constraints coming from the semantics of discourse connectives, is too powerful (there exist some dependency DAGs which respect these constraints, but which seem to correspond to no felicitous discourse). Finally, SDRT comes near to the appropriate strong generative capacity but still falls short.

This conclusion may sound negative, however we hope this study will shed new light on the constraints which must govern discourse structures and on the geometric properties of the graphs representing discourse structures.

Let us insist on the following point: this paper only focuses on discourse structures. Nothing is said about the processes that are or should be implemented to compute discourse structures from discourses.

The paper is organized as follows. Sections 2, 3 and 4 present the main features of the discourse structures proposed respectively in RST, SDRT and dependency DAGs. Section 5 concerns the strong generative capacity of these three formalisms. Section 6 concludes.

In sections 2 to 4, a reference discourse is used. This discourse, taken from [Asher and Lascarides2003], is given in (1).

(1)a. Fred experienced a lovely evening last night.

b. He had a fantastic meal.

c. He ate salmon.

d. He devoured lots of cheese.

e. He won a dancing competition.

This narrative discourse describes Fred's evening which is elaborated with two subevents, the meal and the dancing competition. The meal is itself elaborated with two courses, salmon and cheese. The discourse relations involved and their arguments are not controversial, they are the following:

- Elaboration holds between the first sentence (1a) and the rest of the discourse (1b-e),

- Narration (called Sequence in RST) holds between the meal and dancing competition sentences, i.e. (1b) and (1e), 
- Elaboration holds between the meal sentence (1b) and the two next sentences, i.e. (1c) and (1d),

- Narration holds between the salmon and cheese sentences, i.e. (1c) and (1d).

Elaboration is a nucleus-satellite relation in RST and is subordinating in SDRT, while Narration is multinuclear in RST and coordinating in SDRT. This fits with the claim that nucleus-satellite/subordinating and multinuclear/coordinating are terminological variants.

\section{RST}

RST is a theory which dates back twenty years and which has been widely used in descriptive or computational linguistics as well as in NLP (both for text understanding and text generation). Therefore, the numerous authors working in this framework do not systematically share the same points of view. It is not in the scope of this paper to present the various points of view on RST, see [Taboada and Mann2006a] and [Taboada and Mann2006b] for a review. Thus, we limit ourselves in the next section to a particular interpretation of RST, that of Marcu which has had a strong impact in discourse analysis [Marcu2000a] and discourse annotation [Carlson et al.2003]. However, we discuss in Section 2.2 one of the issues which is debated within the RST community, namely the Nucleus/Satellite distinction.

\subsection{Graphical representations and predicate-argument relations}

The original graphical representation proposed in [Mann and Thompson1987] for discourse structures is illustrated in Figure 1, which shows the discourse structure for (1). In this diagram, the representation of the $i t h$ sentence is noted $\pi_{i}$. This notation comes from SDRT and not from RST where the notation $C_{i}$ is preferred, see for example [Egg and Redeker2007] (this volume). This difference in notation is irrelevant since the representation of sentences is not discussed at all in this paper, which focuses on discourse structures annotated with discourse relations.

[Marcu1996] has proposed graphical representations which are equivalent to the original diagrams, but which do look like trees. For a discourse made up of two sentences (clauses) linked by a discourse relation $R$, the representation is a binary tree: the root is $R$, the edges are labelled $\mathrm{N}$ for Nucleus or $\mathrm{S}$ for Satellite, the (ordered) leaves are the representations of the two sentences. If $R$ is a nucleus-satellite relation, the Nucleus (resp. Satellite) of $R$ is the leaf on the edge labelled N (resp. S); the Nucleus precedes or follows the Satellite. If $R$ is a multi-nuclear relation, both edges are labelled N.

Marcu has also proposed a principle, called "Nuclearity Principle" (or "Compositionality Principle"), which gives the predicate-argument relations when a nucleussatellite (subordinating) relation is embedded in another discourse relation. I extend his principle to give the predicate-argument relations when a multinuclear (coordinating) relation is embedded in another one. This leads to the "Mixed Principle". 


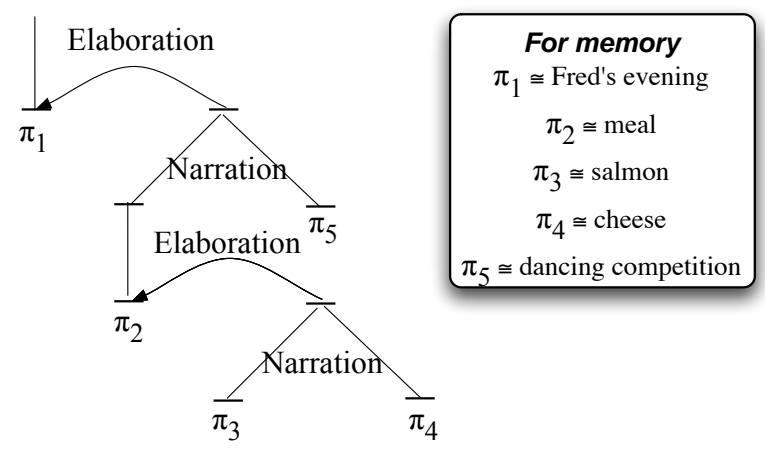

Figure 1: RST diagram for (1) in orthodox style

Mixed Principle: Let $n_{i}$ be a non terminal node in an RST tree whose left (resp. right) daughter is $n_{j}$. The left (resp. right) argument of $n_{i}$ is:

- if $n_{j}$ is a leaf, $n_{j}$

- if $n_{j}$ is a coordinating discourse relation, the sub-tree rooted at $n_{j}$

- if $n_{j}$ is a subordinating discourse relation, the Nucleus argument of $n_{j}$ (recursively given by the Mixed Principle).

This principle is case-based. The two first cases correspond to the standard interpretation of trees used in computer science; the third one corresponds to Marcu's Nuclearity Principle. The RST tree for (1), which must be interpreted with the Mixed Principle, is shown in Figure 2.

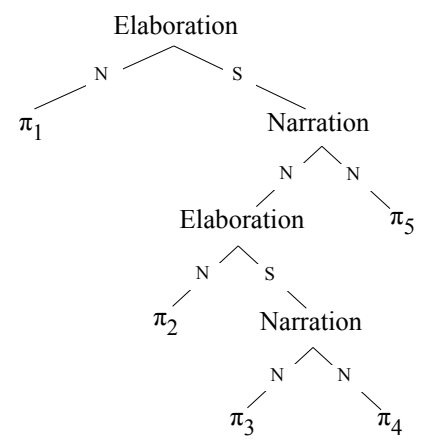

Figure 2: RST tree for (1) in standard style

In this tree, the right (Satellite) argument of the root - the topmost Elaboration - is the sub-tree rooted at the topmost coordinating relation Narration. In this sub-tree, the left argument of the root is $\pi_{2}$ (thanks to the Nuclearity Principle). $\pi_{2}$ is also the left 
argument of the embedded Elaboration. In a nutshell, $\pi_{2}$ is argument of two discourse relations, although it has a single parent in the tree in Figure 2.

\subsection{Nucleus/Satellite distinction}

The criterion put forward by Marcu for the Nucleus/Satellite distinction is the fact that the satellites of a text can be deleted without harming its coherence. He used this criterion for text summarization: his strategy consists in deleting all the Satellites from the original text to keep only the Nuclei [Marcu2000b]. Along the lines of the theory as originally proposed in Mann and Thomson (1988), Marcu considers that only a few discourse relations are multi-nuclear (mainly Sequence, Parallel Contrast, Joint and List). He considers that all the other ones are nucleus-satellite relations, in particular all the relations that can be lexicalized by a subordinating conjunction (the main clause corresponding to the Nucleus, the subordinate clause to the Satellite) [Matthiessen and Thompson1988].

Marcu's position is not approved unanimously within the RST community. On the one hand, his criterion for the Nucleus/Satellite distinction has been criticized, for example, in [Stede2007] where it is proposed that the notion of salience should be taken into account to determine which elements can be qualified as Nuclei according to the context. Moreover, the direct mapping advocated by [Matthiessen and Thompson1988] between the linguistic structure and the type of a discourse relation (i.e. a subordinating conjunction lexicalizes a subordinating relation) has been widely criticized, recently in [Delort2006].

We will see in Section 3.3 that the distinction between coordinating and subordinating relations made in SDRT is also debated.

\section{SDRT}

\subsection{Box representations and graphs for SDRSs}

Originally, [Asher1993] designed SDRT as an extension of DRT - Discourse Representation Theory [Kamp and Reyle1993] - to account for specific properties of discourse. Thus, a discourse structure in SDRT, called an SDRS, gets a box representation à la DRT. In box representations, the distinction between coordinating and subordinating discourse relations is not taken into account. However, the theory, especially the ' logic of information packaging", makes heavy use of this distinction, which is crucial in order to give SDRSs hierarchical structures represented as graphs. Let us introduce this graphical representations.

For a discourse made up of two sentences (clauses) linked by a discourse relation $R$, the nodes of the SDRS graph are the labels $\pi_{1}$ and $\pi_{2}$ of the DRSs giving the semantic representations of the two sentences. They are linked by an arrow labelled by the discourse relation $R$. The arrow "is horizontal with the newer constituent to the right if $R$ is coordinating, while it is vertical (oblique) with the newer constituent below if $R$ is subordinating" [Asher and Vieu2005]. Taking into account the Nucleus/Satellite type of arguments, this means that a horizontal arrow links two Nuclei, while a vertical 
arrow goes from a Nucleus down to a Satellite. It is supposed that the Nucleus of a subordinating relation always precedes the Satellite. We will come back on this simplification in Section 3.4.

In addition to nodes representing sentences (noted $\pi_{i}$ and called "sentence nodes"), SDRS graphs include "scope nodes" (noted $\pi^{\prime}, \pi^{\prime \prime}, \ldots$ ). In the box representation scheme, a scope node labels a sub-SDRS. In the graph scheme, a scope node is linked by lines (and not arrows) to sentence nodes.

Figure 3 illustrates these two representation schemes for the SDRS of (1) (these diagrams are taken from [Asher and Lascarides2003]). The notation $K_{\pi_{i}}$ stands for the DRS representing the ith sentence.

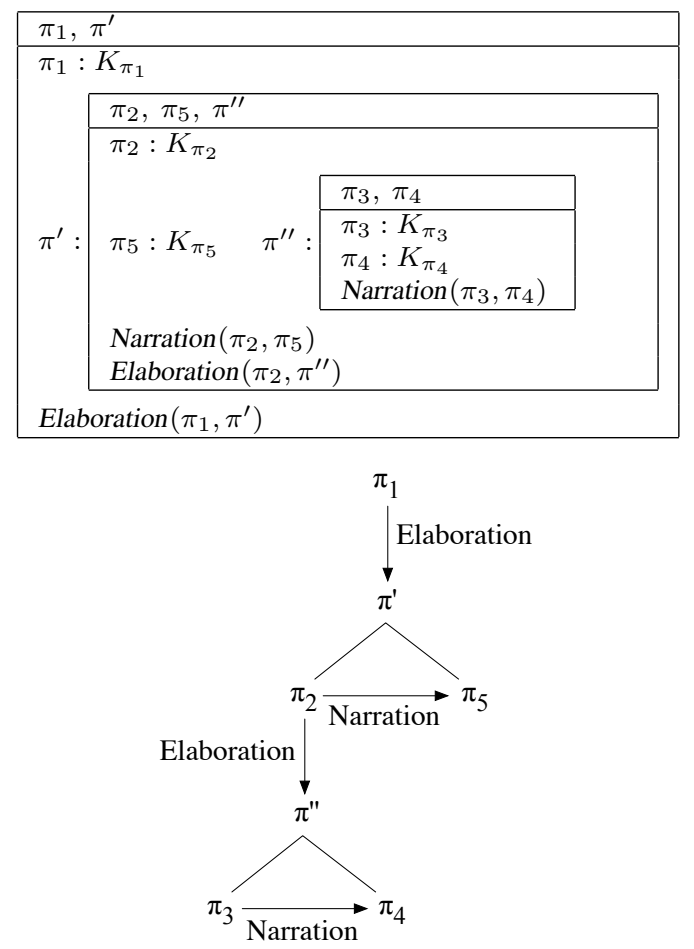

Figure 3: SDRS for (1) in a box representation and as a graph

It should be noted that Narration $\left(\pi_{2}, \pi_{5}\right)$ and Elaboration $\left(\pi_{2}, \pi^{\prime \prime}\right)$ are on an equal footing in the sub-SDRS labelled $\pi^{\prime}$ in the box representation, because no difference is made there between coordinating and subordinating discourse relations. However, this is not the case in the graph: the scope node $\pi^{\prime}$ immediately dominates the two Nuclei $\pi_{2}$ and $\pi_{5}$ of the coordinating relation Narration, while it immediately dominates only the Nucleus $\pi_{2}$ of the subordinating relation Elaboration (it dominates the Satellite $\pi^{\prime \prime}$ but does not immediately dominate it ${ }^{2}$ ). This asymmetry between coordi-

${ }^{2}$ Dominance is the transitive closure of immediate (direct) dominance. 
nating and subordinating relations in SDRS graphs can be considered as equivalent to the asymmetry present in the Mixed Principle in the framework of RST (Section 2.2).

\subsection{Topic nodes}

In addition to sentence and scope nodes, an SDRS can also include "topic nodes" (noted $\pi^{*}, \pi^{* *}, \ldots$ ) which are constructed nodes used for representing the theme of several constituents when this theme is not linguistically realized. These topic nodes are mainly introduced for constituents linked by a coordinating relation. As an illustration, consider the discourse obtained by deleting the first sentence of (1). Its SDRS graph is exactly like the one for (1) except that $\pi_{1}$ is replaced by a topic node $\pi^{*}$ : the content of $\pi^{*}$ is Fred's evening. The introduction of topic nodes is mainly motivated by the Right Frontier Constraint.

\subsection{Right Frontier Constraint}

The notion of "right frontier" was originally proposed by [Polanyi1988]. In an SDRS graph for a discourse with $n$ sentences (clauses), the right frontier contains the node $\pi_{n}$ representing the last sentence, and the sentence and topic nodes which are on the rightmost branch of the graph and dominate $\pi_{n}$. In the graph of Figure 3, the right frontier contains the nodes $\pi_{5}$ and $\pi_{1}$. In the dynamic construction of an SDRS, via an incremental update process, the discourse constituents on the right frontier are the only available sites for attachment of new information. This is known as the "Right Frontier Constraint". Moreover, this constraint states that the antecedent of an anaphoric expression must be ("DRS-accessible") on the right frontier.

The notion of right frontier is therefore crucial in SDRT. As it relies on the distinction between coordinating/subordinating relations, this distinction has been widely discussed [Asher and Vieu2005], [Prevot and Vieu2005]. It is not in the scope of this paper to present all these discussions. Let us just say that these authors consider that a given discourse relation may have only a type by default which can be revised in context. For example, [Asher and Vieu2005] propose that the relation Result is coordinating by default, but that it becomes subordinating in some contexts.

\subsection{Subordinating conjunctions and linear order}

Subordinate conjunctions have been largely ignored in SDRT, in which the focus has been put on inferring the discourse relations which are not lexicalized by a discourse connective. Thereby, preposed subordinate clauses which appear before the main clause, are ignored. Hence, the following simplification: it is assumed that the Nucleus always precedes the Satellite (see the quotation "vertical arrows for subordinating relation with the newer constituent below" in Section 3.1). This simplification is not made in RST: the Satellite of a subordinating relation follows or precedes the Nucleus. As we don't want to anticipate how preposed subordinated clauses should be handled in SDRT, we limit the rest of this study - the goal of which is to compare the generative capacity of RST and SDRT - to the cases where the Satellite of a subordinating relation follows the Nucleus. 


\subsection{Summary on RST and SDRT, discourses in the canonical order}

From this short presentation of RST and SDRT, it should be clear that the two theories do not study exactly the same set of phenomena. To carry on the comparison between the discourse structures they propose, we must limit ourselves to discourses which have been studied in both theories. That is the reason why any discourse including a preposed subordinate clause is put aside, since we have just seen that such subordinate clauses are ignored in SDRT. So the rest of this paper concerns discourses in the "canonical order", i.e. discourses of the form $S_{1}\left(\right.$ Conn $\left._{1}\right) S_{2} \ldots S_{i}\left(\right.$ Conn $\left._{i}\right)$ $S_{i+1} \ldots S_{n}$, which count $n$ sentences, in fact $n$ clauses, noted $S_{i}$, and with no preposed subordinate clauses. The clauses are linked by optional discourse connectives noted Conn $_{i}$ which appear in the initial position of their host sentence. It is assumed that $S_{i}$ includes no discourse connective: this means that cases with multiple discourse connectives are put aside (they are discussed in [Webber et al.2001]). Discourses in canonical order are also supposed to involve no discourse relation such as Attribution. This relation raises difficulties concerning the linear order of its arguments when one of them is embedded in the other one. It is discussed in [Redeker and Egg2006] in the framework of RST and in [Hunter et al.2006] in the framework of SDRT.

The representation of $S_{i}$, whatever it is, is noted $\pi_{i}$. A connector Conn $n_{i}$ lexicalizes a discourse relation noted $R_{i}$. If Conn $n_{i}$ is not present, it is all the same assumed that there is a discourse relation $R_{i}$. In fact, there could be several discourse relations between two constituents if they are of the same type, i.e. either all coordinating or all subordinating. This is authorized in SDRT but not in RST. It can easily be authorized in RST without any drastic change: a node noted $R_{i} / R_{i}^{\prime}$ in an RST tree can be used to indicate that the two relations $R_{i}$ and $R_{i}^{\prime}$ hold between two constituents. As $R_{i}$ and $R_{i}^{\prime}$ are of the same type, there is no problem with the Nucleus/Satellite type of their arguments. In SDRS graphs, a horizontal arrow can be labelled $R_{i} / R_{i}^{\prime}$ if $R_{i}$ and $R_{i}^{\prime}$ are both coordinating, and a vertical arrow can be labelled $R_{i} / R_{i}^{\prime}$ if $R_{i}$ and $R_{i}^{\prime}$ are both subordinating.

It is clear that the discourse structures proposed in RST and SDRT are quite different, however these two theories share the fact that they rely upon discourse relations and the distinction Nucleus/Satellite or coordinating/subordinating. Do they rely upon the same set of discourse relations and do they give them the same type? The answer to these two questions is roughly affirmative. In fact, [Asher1993] started from the discourse relations proposed in [Mann and Thompson1988] and even if there exist few differences in the set of discourse relations used in RST and SDRT, they are not relevant for the study proposed here. Concerning the type given to a discourse relation, it is the same in RST and SDRT in most cases. The well-known exception is the relation Result which is subordinating in RST and coordinating (by default) in SDRT. As a consequence, no example involving Result will be presented in the rest of this paper.

We are now going to present another mode of representation for discourse structures, which is inspired by dependency grammars and which doesn't rely upon the distinction between coordinating and subordinating relations. 


\section{Discourse dependency DAGs}

Among dependency grammars, the most famous theory is probably MTT (MeaningText Theory) [Mel'cuk1988], designed for sentence generation but adapted to sentence analysis by [Kahane2001]. It involves three levels of representation: semantic, syntactic and morphologic. We propose below an extension of the semantic level for discourse, and compare the discourse structures obtained to those proposed in RST and SDRT.

The core of the semantic level in MTT is a directed labelled graph in which nodes are "semantemes", either lexical or grammatical. A lexical semanteme represents a meaning of a word (e.g. 'bake1' (a potato) and 'bake2' (a cake) are two lexical semantemes for the verb bake). A semanteme is viewed as a predicate which is linked to its arguments (if any) by arrows pointing towards them. These arrows are labelled with numbers which distinguish the arguments.

For discourse, it can be stated that discourse relations are semantemes when they are lexicalized by a discourse connective. In this perspective, a discourse relation corresponds to the meaning of a discourse connective or to one of its meanings. By extrapolation, discourse relations which are not lexicalized are also considered as semantemes. Two sentences linked by a discourse relation $R$ receive thus the same representation as in RST, namely a binary tree whose root is $R$ and whose leaves are the sentence representations. However, there is a crucial difference between semantic dependency graphs and RST representations, namely the tree structure of the graphs: a semantic dependency graph is not always tree shaped, while RST representations are compulsorily tree shaped (Section 2.1). This difference comes from the way predicate-argument relations are computed. In semantic dependency graphs, whether they represent a sentence as in MTT or a discourse in the MTT extension proposed here, predicate-argument relations are computed in a simple and standard way: the arguments of a predicate (e.g. a discourse relation) are always its daughters. There is no equivalent to the Nuclearity principle used in RST ( Section 2.1). For example, the dependency graph for (1) - in fact a DAG, see below - is shown in Figure 4. In this graph, $\pi_{2}$ has two parents, which straightforwardly translates the fact that $\pi_{2}$ is argument of two discourse relations. On the other hand, it must be remembered that this fact is not graphically visible in the RST tree for (1) (see Figure 2); it requires the help of the Nuclearity Principle.

Convention: In this paper, any RST tree must be interpreted with the Mixed Principle, any dependency graph with the standard interpretation. To avoid confusion, edges are graphically represented as lines in RST trees and as arrows in semantic dependency graphs.

What are the constraints which govern dependency graphs representing discourse structures?

First, it is assumed that they are acyclic. Therefore, these dependency graphs are DAGs (Directed Acyclic Graphs). In these DAGs, it is assumed that the leaves, projected on a horizontal line, are ordered, as it is the case of the leaves of an RST tree. It is also assumed that any non terminal node has exactly two daughters, which comes from the fact that a non terminal node is a discourse relation with two arguments (see note 1).

Second, from our knowledge of the semantics of discourse connectives, two (minimal) constraints, noted $C_{1}$ and $C_{2}$, can be postulated for discourses in the canonical 


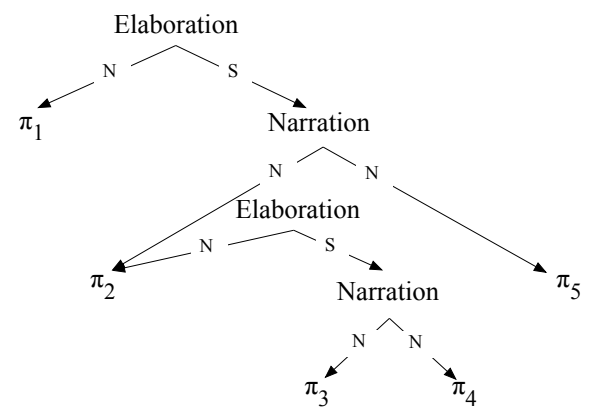

Figure 4: Dependency graph for (1)

order (in particular, without preposed subordinate clauses, see Section 3.5) of the form $S_{1}\left(C_{o n n_{1}}\right) S_{2} \ldots S_{i}\left(C_{\text {Conn }}\right) S_{i+1} \ldots S_{n}$. Constraint $C_{1}$ states that the first argument of a discourse connective Conn $n_{i}$ is on the left of Conn $n_{i}$. Constraint $C_{2}$ states that a sentence $S_{i+1}$ introduced by a discourse connective $C_{o n n}$ is under the semantic scope of this connective. By extrapolation, I postulate that these two constraints also hold when a discourse relation $R_{i}$ is not lexicalized by a discourse connective. These constraints are formulated as follows in semantic dependency DAGs.

Constraint $C_{1}$ : the first argument of $R_{i}$ is the representation of a text span which appears on the left of $\left(\right.$ Conn $\left._{i}\right) S_{i+1}$.

Constraint $C_{2}$ : the second argument of $R_{i}$ is the representation of a text span which starts at $\pi_{i+1}$ (this text span can be reduced to $\pi_{i+1}$ ). In terms of dominance, $C_{2}$ means that $R_{i}$ dominates $\pi_{i+1}$.

Let us show that constraints $C_{1}$ and $C_{2}$ are respected both in RST and SDRT, which is not a surprise since they are minimal. In RST, the "adjacency principle" is postulated. It states that the arguments of a discourse relation expressed through a discourse connective are given by continuous text spans which are adjacent to the discourse connective [Mann and Thompson1987]. The adjacency principle is also postulated when $R_{i}$ is not lexicalized. This principle makes no distinction between the first and second argument of a discourse relation. More precisely, it is equivalent to constraints $C_{1}^{\prime}$ and $C_{2}$, in which $C_{1}^{\prime}$ mirrors $C_{2}$ ( $C_{1}^{\prime}$ is thus a constraint which is stronger than $C_{1}$ ).

Constraint $C_{1}^{\prime}$ : the first argument of $R_{i}$ is the representation of a text span which ends at $\pi_{i}$ (this text span can be reduced to $\pi_{i}$ ). In terms of dominance, $C_{1}^{\prime}$ means that $R_{i}$ dominates $\pi_{i}$.

Constraints $C_{1}^{\prime}$ and $C_{2}$ are used in [Egg and Redeker2007] (this volume) for underspecified discourse representations in the framework of RST. For a discourse in the canonical order with $n$ sentences, the underspecified representation they propose is given in Figure 5 (a dotted line represents dominance, a solid line immediate domi- 
nance). This underspecified representation respects exactly constraints $C_{1}^{\prime}$ and $C_{2}$.

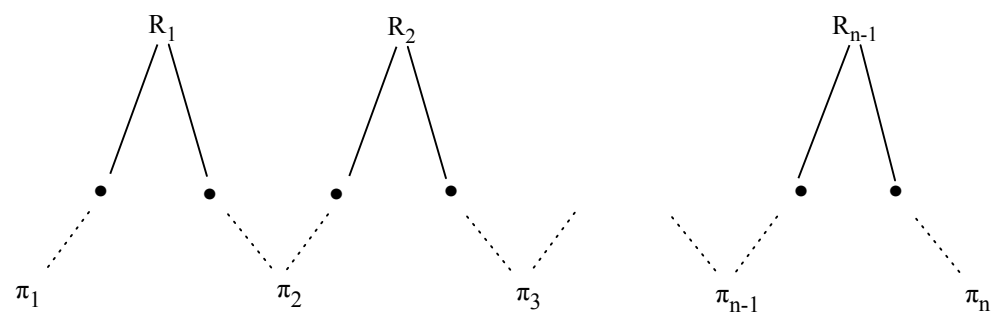

Figure 5: Underspecified RST representation from [Egg and Redeker2007]

In SDRT, constraints $C_{1}^{\prime \prime}$ (below) and $C_{2}$ can be inferred from the incremental updating process. In a simplified way, when dealing with the current sentence $S_{i+1}$, the underspecified condition ? $R\left(\alpha, \pi_{i+1}\right)$ holds, in which ?R is a discourse relation variable, which will be specified as $R_{i}$ in our notation, and $\alpha$ is an attachment site which must be on the right frontier of the SDRS graph representing the left-context of $\left(\right.$ Conn $\left._{i}\right) S_{i+1}$ (Section 3.3). Therefore, a constraint stronger than $C_{1}$ holds for the first argument of $R_{i}$, namely $C_{1}^{\prime \prime}$.

Constraint $C_{1}^{\prime \prime}$ : the first argument of $R_{i}$ is a text span which appears on the right frontier of the SDRS graph representing the left context of $\left(\mathrm{Conn}_{i}\right) S_{i+1}$.

For the second argument of $R_{i}$, the underspecified condition $? R\left(\alpha, \pi_{i+1}\right)$ states that $\pi_{i+1}$ is the second argument of $R_{i}$. However, later on in the non-monotonous updating process, this condition may be revised so that $R_{i}$ only dominates $\pi_{i+1}$. This means that $\mathrm{C}_{2}$ is also respected in SDRT.

In conclusion, dependency graphs representing discourse structures are ordered DAGs, in which non-terminal nodes are discourse relations with two daughters. The predicate-argument relations respect a heavy constraint $C_{2}$ on the right argument and a weaker constraint $C_{1}$ on the left argument. RST and SDRT also respect $C_{2}$ for the second (right) argument, while these discourse theories respect respectively $C_{1}^{\prime}$ and $C_{1}^{\prime \prime}$ for the first (left) argument, these constraints being stronger than $C_{1}$.

From this data, one can expect dependency DAG formalism to be more powerful in strong generative capacity than RST and SDRT. This will indeed be shown in Section 5. Constraints $C_{1}^{\prime}$ and $C_{1}^{\prime \prime}$ cannot be directly compared without taking into account other RST or SDRT constraints (e.g. the constraint stating that RST structures must be tree shaped). However, it will be shown in Section 5 that RST is less powerful in strong generative capacity than SDRT.

Let us underline the following point: the only constraints which hold on discourse dependency DAGs are $C_{1}$ and $C_{2}$ which don't involve the coordinating/subordinating distinction, which is so crucial in RST and SDRT. The next section will examine the consequences of this fundamental difference.

To illustrate the constraints on dependency DAGs, let us examine what are the possible DAGs for discourses in the canonical order with three sentences, a case which 
will be thoroughly studied in Section 5 . The possible DAGs have three ordered leaves, $\pi_{1}<\pi_{2}<\pi_{3}$, two non terminal nodes $R_{1}$ and $R_{2}$. The heavy constraint $C_{2}$ means that the second argument of $R_{1}$ must start at $\pi_{2}$, and that $\pi_{3}$ is compulsorily the second argument of $R_{2}$. The weaker constraint $C_{1}$ means that $\pi_{1}$ is compulsorily the first argument of $R_{1}$. These constraints leave us with four possible non-labelled DAGs ${ }^{3}$, which are shown in Figure 6. Two of them are non tree shaped (one in which $\pi_{1}$ has two parents, the other one in which $\pi_{2}$ has two parents), the two others are tree shaped.

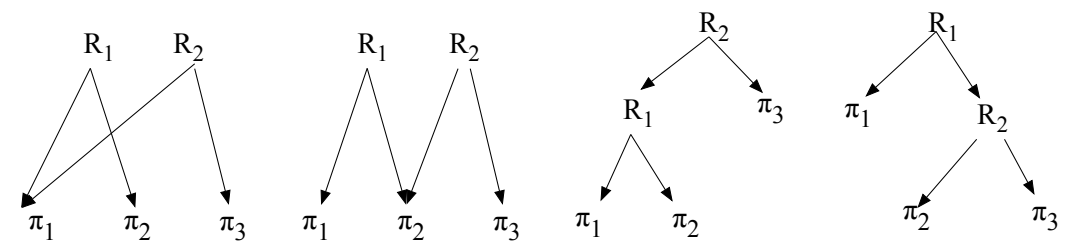

Figure 6: Non-labelled DAGs for a discourse with three sentences in the canonical order, respecting constraints $C_{1}$ and $C_{2}$

A crucial point is that constraint $C_{2}$ excludes the non tree shaped DAG in which $\pi_{3}$ has two parents, namely the DAG in Figure 7. This DAG is excluded since the second argument of $R_{1}$ does not start at $\pi_{2}$ (in other words $R_{1}$ does not dominate $\pi_{2}$ ), and so constraint $C_{2}$ is not respected. There seems to exist no felicitous discourse whose structure is the DAG in Figure 7. As explained in [Danlos2004], this is justified on psycho-linguisitic grounds: what would be a discourse in which the second sentence is not linked at all to the first one? $?^{4}$

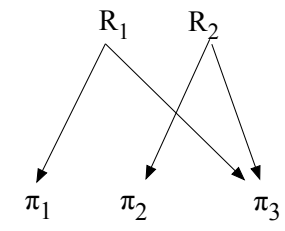

Figure 7: Non-labelled DAG which does not respect constraint $C_{2}$

Summary of the representations for discourse structures: Taking as illustration the discourse in (1), we have examined three representations for discourse structures: RST trees, SDRT graphs and dependency DAGs.

\footnotetext{
${ }^{3}$ Non-labelled graphs do not take into account the labels $(\mathrm{N}$ or $\mathrm{S})$ on the edges.

${ }^{4}$ The link between the first two clauses of a discourse can be given by a third sentence, as in (i) below in which $S_{3}$ establishes a joint link between $S_{1}$ and $S_{2}$ through its plural subject. See also (ii) in which the first two sentences establish the setting for the third sentence. These discourses must be orally uttered with a special intonation.

(i) It is raining. Fred arrived late. These two facts irritated Mary.

(ii) The sun was shining. Nice music was playing on the radio. Fred woke up in a good mood that day.
} 
We are going to compare the strong generative capacity of these three formalisms.

\section{$5 \quad$ Strong generative capacity}

To compare the strong generative capacity of the three formalisms under study, I start with quite a simple case of discourses in the canonical order, namely discourses with three sentences (clauses) noted here as $S_{1}\left(\right.$ Conn $\left._{a}\right) S_{2}\left(\right.$ Conn $\left._{b}\right) S_{3}$. Their discourse structures involve three sentence representation nodes noted $\pi_{i}$ and two discourse relations noted $R_{a}$ and $R_{b}$. The methodology is the following: I begin with RST trees, since RST is the most constraining formalism. Next I move to semantic dependency DAGs, since this formalism is the least constraining one. This will allow me to situate SDRT in between these two formalisms.

\subsection{RST trees and their equivalents in the other representations}

For discourses in the canonical order of the form $S_{1}\left(\right.$ Conn $\left._{a}\right) S_{2}\left(C_{\text {onn }}\right) S_{3}$, RST trees must have three ordered leaves $\left(\pi_{1}<\pi_{2}<\pi_{3}\right)$ and two internal nodes $\left(R_{a}\right.$ and $R_{b}$ ). To respect the tree structure, one internal node must be the daughter of the other one. Therefore, there exist only two non-labelled binary trees ${ }^{5}$, namely either $R_{b}\left(R_{a}\left(\pi_{1}, \pi_{2}\right), \pi_{3}\right)$ or $R_{a}\left(\pi_{1}, R_{b}\left(\pi_{2}, \pi_{3}\right)\right)$. These two trees lead to eight RST trees with the edges labelled. First, the four cases with an embedded subordinating discourse relation are examined, next the four other cases - with an embedded coordinating relation - are examined.

Cases with an embedded subordinating relation: In Table 1, the first row shows the four RST trees with an embedded satellite-nucleus relation, namely (Ia)-(IVa). The second row shows the equivalent dependency DAGs, namely (Ib)-(IVb). None of these DAGs is tree shaped. This comes from the fact that the Nuclearity Principle is involved to compute the predicate-argument relations in the RST trees (Ia)-(IVa) since the embedded relation is subordinating. For example, in (Ia), the Nucleus of $R_{b}$ is $\pi_{1}$, which is also the Nucleus of $R_{a}$; hence the dependency DAG (Ib), in which $\pi_{1}$ has two parents.

The third row in Table 1 shows the equivalent SDRT graphs. Let us describe the SDRT graph in (Ic) with the help of the predicate-argument relations visible in (Ib): starting from $\pi_{1}$, there is a vertical arrow pointing towards $\pi_{2}$ and labelled with the subordinating relation $R_{a}$. Next, from $\pi_{1}$, there is a horizontal arrow pointing towards $\pi_{3}$ and labelled with the coordinating relation $R_{b}$. The scope label $\pi^{\prime}$ immediately dominates $\pi_{1}$ and $\pi_{3}$, and dominates $\pi_{2}$.

The four discourse structures (I)-(IV) given in Table 1 can all be linguistically realized in felicitous discourses, for example in the discourses given in (2).

(2) a. Fred was badly sick last week. He had a bad flu. However, he is in good shape this week

Structure (I) with $R_{a}=$ Elaboration and $R_{b}=$ Contrast

\footnotetext{
${ }^{5}$ As explained in note 3, non-labelled graphs don't take into account the labels $\mathrm{N}$ and $\mathrm{S}$ on the edges, that is the coordinating vs subordinating nature of discourse relations.
} 


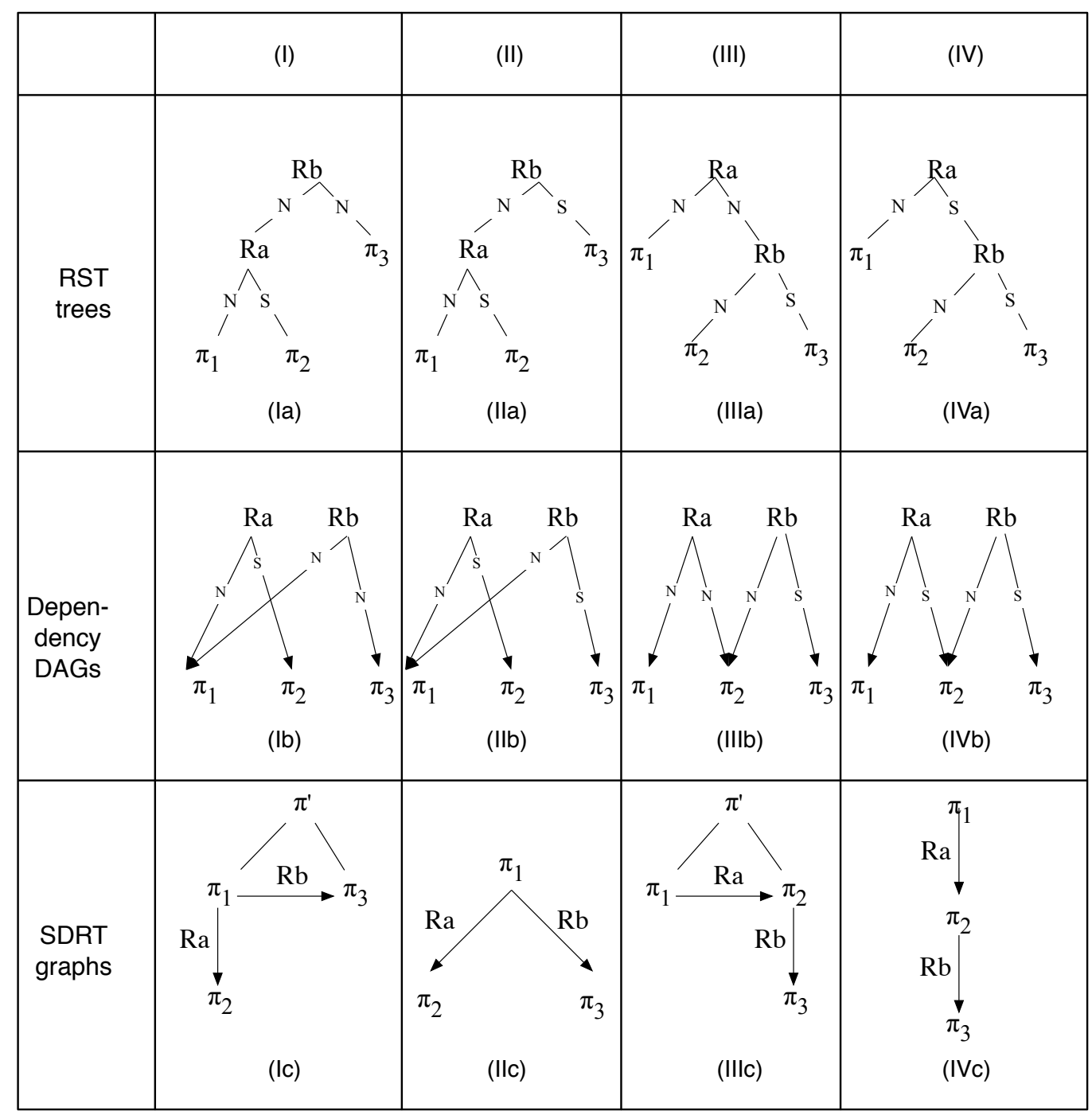

Table 1: RST trees for $S_{1}\left(\right.$ Conn $\left._{a}\right) S_{2}\left(\right.$ Conn $\left._{b}\right) S_{3}$ with an embedded subordinating relation, and their equivalent dependency DAGs and SDRT graphs. 
b. Fred is badly sick. He probably has the flu. He took a walk in the rain yesterday. Structure (II) with $R_{a}=$ Elaboration and $R_{b}=$ Explanation

c. Fred is in good shape. However, Mary is badly sick. She has a bad flu. Structure (III) with $R_{a}=$ Contrast and $R_{b}=$ Elaboration

d. Fred was in a foul mood. He hadn't slept well that night. His electric blanket hadn't worked. ${ }^{6}$

Structure $(I V)$ with $R_{a}=$ Explanation and $R_{b}=$ Explanation

Cases with an embedded coordinating relation: In Table 2, the first row shows the four RST trees with an embedded multi-nuclear relation, namely (Va)-(VIIIa). The second row shows the equivalent dependency DAGs, namely (Vb)-(VIIIb), which are all tree shaped. The RST trees and dependency DAGs are quite similar: graphically, they differ only by the edges which are lines in RST trees and arrows in dependency DAGs, according to the convention presented in Section 4 . This similarity comes from the fact that the predicate-argument relations in the RST trees (Va)-(VIIIa) are computed in the standard way (i.e. without involving the Nuclearity Principle). For example, in (Va), the Nucleus of $R_{b}$ is the sub-tree rooted at $R_{a}$, hence the dependency DAG $(\mathrm{Vb})$. The third row in Table 2 shows the equivalent SDRT graphs. In these graphs, the topic nodes are omitted for the sake of simplicity: the scope nodes are supposed to play their roles on the right frontier for the attachment of new information.

The four discourse structures (V)-(VIII) given in Table 2 can all be linguistically realized in felicitous discourses, for example in (3).

(3) a. Fred ate a big salmon. He also devoured a lot of cheese. On the other hand, Mary missed her diner.

Structure $(V)$ with $R_{a}=$ Parallel/Narration ${ }^{7}$ and $R_{b}=$ Contrast

b. Fred ate a big salmon. He also devoured a lot of cheese. This was a fantastic meal.

Structure (VI) with $R_{a}=$ Parallel/Narration and $R_{b}=$ Comment $^{8}$

c. Fred missed his diner. On the other hand, Mary ate a big salmon. She also devoured a lot of cheese.

Structure (VII) with $R_{a}=$ Contrast and $R_{b}=$ Parallel/Narration

d. Fred had a fantastic meal. He ate a big salmon. He also devoured a lot of cheese.

Structure (VIII) with $R_{a}=$ Elaboration and $R_{b}=$ Parallel/Narration

In conclusion, for discourses in the canonical order of the form $S_{1}\left(\right.$ Conn $\left._{a}\right) S_{2}$ $\left(\right.$ Conn $\left._{b}\right) S_{3}$, RST allows exactly eight discourse structures. These eight RST trees correspond to dependency DAGs and SDRT graphs which are authorized in these two formalisms. They can all be linguistically realized in felicitous discourses.

\footnotetext{
${ }^{6}$ This discourse is taken from [Hobbs 1979].

${ }^{7}$ The notation $R_{a}=$ Parallel/Narration has been introduced in Section 3.5. It means that the two coordinating relations Parallel and Narration can be inferred to link $\pi_{1}$ and $\pi_{2}$.

${ }^{8}$ Comment is subordinating both in RST and SDRT.
} 


\begin{tabular}{|c|c|c|c|c|}
\hline & (V) & (VI) & (VII) & (VIII) \\
\hline $\begin{array}{l}\mathrm{RST} \\
\text { trees }\end{array}$ & 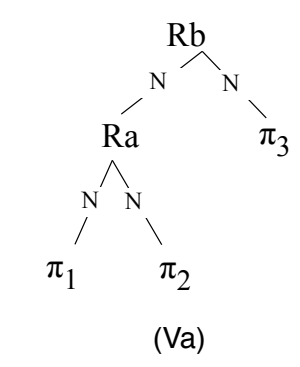 & 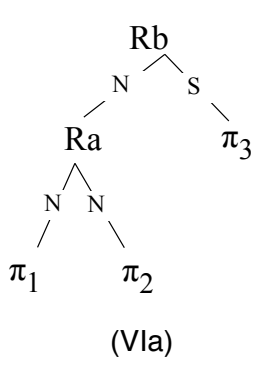 & 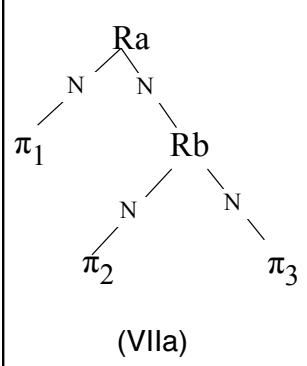 & 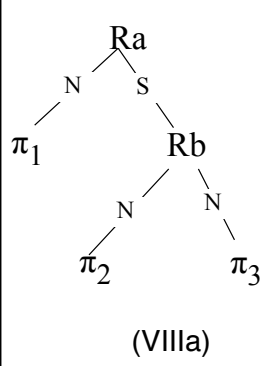 \\
\hline $\begin{array}{l}\text { Depen- } \\
\text { dency } \\
\text { DAGs }\end{array}$ & $\pi_{\pi_{1}}^{\mathrm{Rb}} \overbrace{(\mathrm{Vb})}^{\mathrm{Ra}}$ & 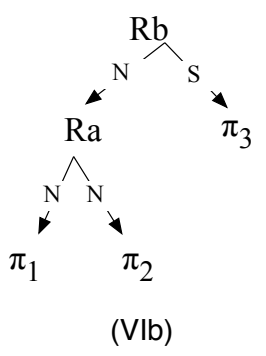 & $\pi_{1} \pi^{\mathrm{Ra}}$ & 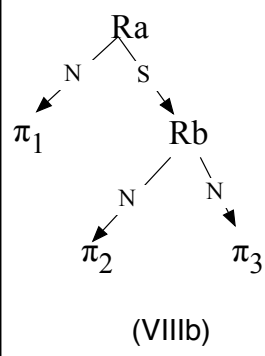 \\
\hline $\begin{array}{l}\text { SDRT } \\
\text { graphs }\end{array}$ & 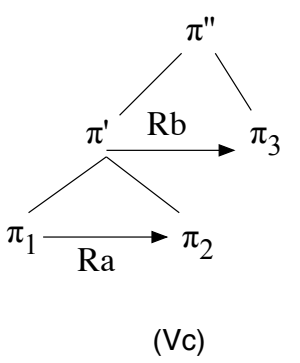 & 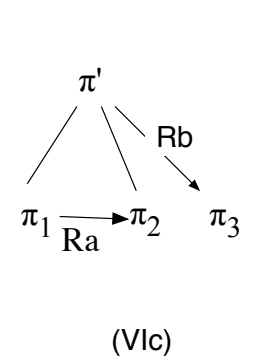 & $\pi_{1} \stackrel{\mathrm{Ra}}{\pi_{\mathrm{Rb}}} \pi_{(\mathrm{VIIc})}^{\pi^{\prime \prime}} \pi_{3}$ & 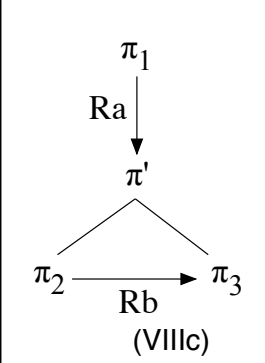 \\
\hline
\end{tabular}

Table 2: RST trees for $S_{1}\left(\right.$ Conn $\left._{a}\right) S_{2}\left(\right.$ Conn $\left._{b}\right) S_{3}$ with an embedded coordinating relation, and their equivalent dependency DAGs and SDRT graphs. 


\subsection{Dependency DAGs without any equivalent RST tree}

We have shown in Section 4 that there are four non-labelled DAGs for discourses in the canonical order of the form $S_{1}\left(\right.$ Conn $\left._{a}\right) S_{2}\left(\right.$ Conn $\left._{b}\right) S_{3}$, respecting constraints $C_{1}$ and $C_{2}$. When the edges are labelled, sixteen DAGs are obtained (four for each non-labelled DAG). Eight of these DAGs have already been examined in the previous section: DAGs (Ib)-(VIIIb) in Tables 1 and 2 with an equivalent RST tree. We are left with the eight other DAGs without any equivalent RST tree, which thus correspond to discourse structures excluded in RST. We are going to examine whether these discourse structures are also excluded in SDRT or not, and study their linguistic realization. We start with the four non tree shaped DAGs.

\subsubsection{Non tree shaped DAGs without any equivalent RST tree}

Non tree shaped DAGs in which $\pi_{1}$ has two parents: DAGs (IXb) and (Xb) in Table 3 differ from DAGs (Ib) and (IIb) in Table 1 by the fact that $R_{a}$ is coordinating (and not subordinating). Hence the impossibility of obtaining RST trees (interpreted with the Mixed Principle) with the same predicate-argument relations.

These DAGs convert into the SDRT graphs (IXc) and (Xc), which are excluded by the Right Frontier Constraint (Section 3.3): $\pi_{3}$ cannot be attached to $\pi_{1}$ which is not on the right frontier.

Non tree shaped DAGs in which $\pi_{2}$ has two parents: DAGs (XIb) and (XIIb) in Table 3 differ from DAGs (IIIb) and (IVb) in Table 1 by the fact that $R_{b}$ is coordinating (and not subordinating). In structure (XII), it is assumed that $R_{a} \neq R_{b}$. In other words, this discourse structure does not involve a unique discourse relation linking three constituents.

SDRT graph (XIc) is excluded by the "Continuing Discourse Pattern Constraint" which states that "coordinated constituents of a substructure must behave in a homogeneous fashion with respect to a dominating constituent", [Asher and Vieu2005] ${ }^{9}$. In the non-monotonous updating process of SDRT, graph (XIc) is compulsorily transformed into graph (VIIIc) in Table 2, in which the two coordinated constituents are dependent on $\pi_{1}$.

On the other hand, SDRT graph (XIIc) is not excluded by any constraint.

Remark on the arborescence of SDRT graphs and their projectivity: SDRT graphs never look like trees since they contain horizontal arrows (for coordinating relations). Nevertheless, one can disregard these horizontal arrows and examine the arborescence of SDRT graphs focusing on the relations between a mother and a daughter coming from a subordinating relation (graphically a vertical or oblique arrow) or a scope relation (graphically an oblique line). In this perspective, SDRT graphs (Ic)-(IXc) look like trees with a single root and a single parent for each node. However, this is not the case for (Xc)-(XIIc): in each of these graphs, one node has two parents $-\pi_{1}$ in (Xc), $\pi_{2}$ in

\footnotetext{
${ }^{9}$ A similar constraint has been put forward in syntax for coordination: [Sag et al.1985], for example, state that two constituents can be coordinated only if they have the same syntactic function.
} 


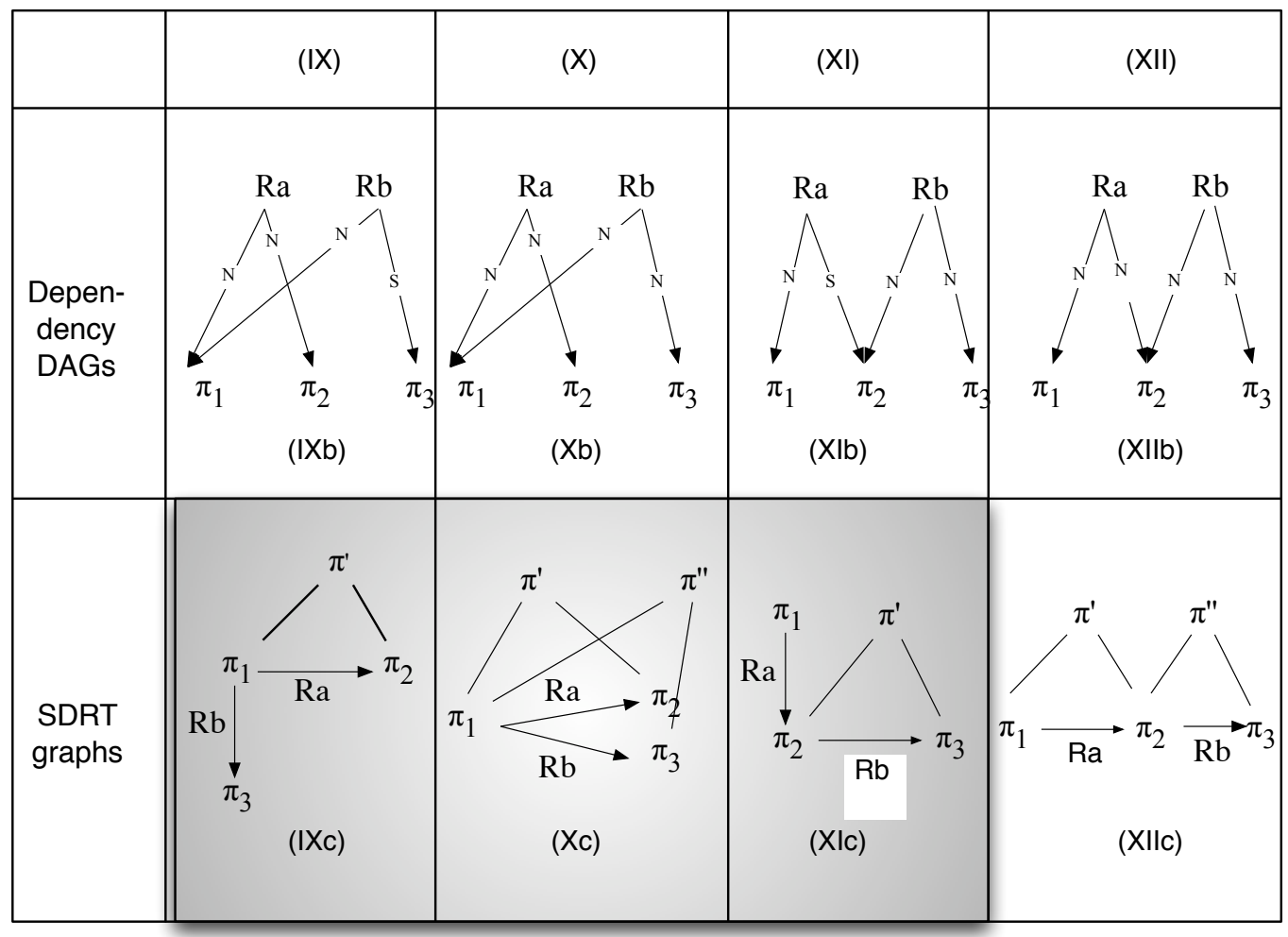

Table 3: Non tree shaped dependency DAGs without any equivalent RST tree, and their equivalent SDRT graphs (on a shaded background for those which are excluded by the theory) 
(XIc) and (XIIc). The fact that (XIIc) is not excluded means that an SDRT graph can be non tree shaped.

SDRT graph (IXc) is tree shaped (disregarding horizontal arrows) but it doesn't respect the Right Frontier Constraint. Let us show that this SDRT tree is not "projective" 10 . The notion of projectivity has been introduced in dependency grammars for syntax. First a definition: in a tree, the (maximal) projection of a node $x$, noted $\operatorname{Proj}(x)$, is the set of nodes dominated by $x, x$ included. A syntactic dependency tree for a sentence is projective iff all the projections of words are continuous segments of the sentence [Lecerf1961]. Lecerf proved that a dependency tree is projective iff dependencies never cross each other and no dependency covers the root. As an illustration, if $w_{1}, w_{2}, w_{3}$, and $w_{4}$ are four words occurring in a projective dependency tree with the linear order $w_{1}<w_{2}<w_{3}<w_{4}$, then it is not possible that $w_{1}$ be linked to $w_{3}$ and $w_{2}$ to $w_{4}$ (such a case is known as "crossing dependencies"). The notion of projectivity can be straightforwardly extended to SDRT trees (i.e. SDRT graphs which are tree shaped disregarding horizontal arrows). The reader can check, for example, that the SDRT graph for (1) in Figure 3 is a projective tree. The SDRT graphs (Ic)-(VIIIc) are also projective trees. On the other hand, this is not the case for (IXc) which is not projective: $\operatorname{Proj}\left(\pi_{1}\right)=\left\{\pi_{1}, \pi_{3}\right\}$ does not form a continuous segment since there is $\pi_{2}$ which intervenes between $\pi_{1}$ and $\pi_{3}$. In summary, (Ic)-(VIIIc) are projective trees and they respect the Right Frontier Constraint, while (IXc) is not projective and doesn't respect the Right Frontier Constraint. More generally, it is possible to show that an SDRT tree is projective iff it respects the right frontier constraint (Sylvain Kahane, pc) ${ }^{11}$.

We are now going to examine how the discourse structures (IX)-(XII) given in Table 3 are linguistically realized. We start with structure (IX), which raises questions on the status of anaphoric relations in discourse structures.

Linguistic realization of (IX): The discourse (4) involves an anaphoric link between an indefinite NP (a salmon) in the first sentence and a definite NP in the third sentence (the salmon). It can be given structure (IX), represented as the dependency DAG (IXb), with $R_{a}=$ Parallel/Narration and $R_{b}=$ Elaboration/Comment. This discourse is felicitous, and numerous examples following this pattern, in which the third sentence elaborates/comments an entity occurring in the first sentence, can be found in corpora.

(4) Fred ate a big salmon. He also devoured a lot of cheese. The salmon came from Norway.

As structure (IX) does not correspond to any RST tree (because of the Mixed Principle) and is excluded in SDRT (because of the Right Frontier Constraint), other analyses for (4) are proposed in these discourse theories. In the framework of RST, [Egg and Redeker2007] (this volume) would give (4) the tree shaped structure (VIa) in Table 2, with again $R_{a}$ $=$ Parallel/Narration and $R_{b}=$ Elaboration/Comment. In this tree, the Nucleus of $R_{b}$ is

\footnotetext{
${ }^{10}$ I thank Sylvain Kahane for drawing my attention to projectivity issues.

${ }^{11}$ This rule is valid only for SDRT trees such that the Nucleus of any subordinating relation occurs before the Satellite. This is the case for the SDRT trees studied here, which represent discourses in the canonical order (Section 3.5).
} 
the sub-tree rooted at $R_{a}$. This means that the anaphoric link between $a$ salmon and the salmon is ignored in the discourse structure. More generally, Egg and Redeker claim that "anaphora can create relations between sentences that are not directly linked by discourse structure". This position is not adopted by [Wolf and Gibson2005]: although these authors work in the RST framework, they would give (4) structure (IX) and use such examples as evidence against the arborescence of discourse structures.

In the framework of SDRT, the anaphoric definite expression the salmon in (4) violates the constraint stating that the antecedent of an anaphoric expression must be on the right frontier (Section 3.3). However, [Asher2007] (this volume) claims that such a definite NP, which has presuppositional content, is accommodated with the following consequence: the referent for a salmon is introduced in the topic $\pi^{*}$ of $\pi_{1}$ and $\pi_{2}$ (see Section 3.2 for the notion of topic). $\pi^{*}$ is on the right frontier and $\pi_{3}$ is attached to $\pi^{*}$. The SDRT graph for (4) is shown in Figure 8, with $R_{a}=$ Parallel/Narration and $R_{b}=$ Elaboration/Comment. It respects the Right Frontier Constraint both for the attachment of $\pi_{3}$ and for the antecedent of the salmon. As explained in Section 5.2, topic nodes are omitted in the diagrams of Table 2 for the sake of simplicity. If it were not the case, the diagram (VIc) in Table 2 would be replaced by the one in Figure 8. This amounts to saying that [Asher2007] gives (4) structure (VI) (disregarding topic nodes), which is the structure advocated in [Egg and Redeker2007] in the framework of RST.

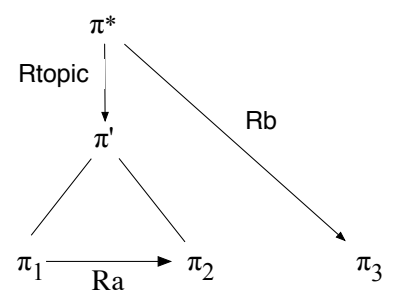

Figure 8: SDRT graph proposed in [Asher2007] for discourse (4)

To sum up, (4) is given either structure (VI) or (IX) according to the positions adopted for the status of anaphoric relations in discourse structures.

We have seen that (IXc) is not projective. In syntax, it is well-known that most structures for English (or French) sentences are projective, but not all of them. If (4) is given structure (IX), then it can be stated that most structures for English (or French) discourses are projective, but not all of them.

What can be said about crossing dependencies in discourse? Consider discourses with four sentences (clauses) in which the third sentence elaborates an entity occurring in the first one (or elaborates the first one) and the fourth sentence elaborates an entity occurring in the second one (or elaborates the second one). Such discourses, which have been examined by [Stede1999], [Wolf and Gibson2005] and [Egg and Redeker2007] (this volume), are illustrated in (5), with the anaphoric links a big salmon/the salmon and $a$ lot of cheese/the cheese.

(5) Fred ate a big salmon. He also devoured a lot of cheese. The salmon came from 
Norway. The cheese came from France.

If anaphoric links are taken into account in discourse structure - Wolf and Gibson's position - then the dependency DAG for (5) includes crossing dependencies, namely Elaboration $\left(\pi_{1}, \pi_{3}\right)$ and Elaboration $\left(\pi_{2}, \pi_{4}\right)$. On the other hand, Egg and Redeker (this volume) and Asher (pc.) would give (5) a structure which doesn't reflect the anaphoric links ${ }^{12}$.

In conclusion, a definite anaphoric NP can have its antecedent a priori anywhere in its left context (as long as binding constraints are respected). Taking into account anaphoric links in discourse structures raises a problem for the arborescence of RST structures and for the Right Frontier Constraint in SDRT, and leads to crossing dependencies in dependency DAGs. Another solution, advocated by Egg in RST and Asher in SDRT, consists in not systematically representing anaphoric links in discourse structures. I have no conclusive argument to decide between these two solutions.

Linguistic realization of $(\mathbf{X})$ : Structure $(X)$ in Table 3, in which two coordinating relations have the same constituent as first argument, violates the SDRT Right Frontier Constraint for the attachment of $\pi_{3}$. It turns out that it is hard to imagine an example with this structure. Nevertheless, let us examine the discourse in (6a). It is a priori of structure (X) with also lexicalizing $R_{a}=$ Parallel and next lexicalizing $R_{b}=$ Narration. However, one can argue that the second sentence (Mary did too) is perceived as secondary information, which amounts to demoting the coordinating relation Parallel down to a subordinating relation, and therefore to giving (6a) structure (I) in Table 1 instead of structure $(\mathrm{X})$. We are left with the cases where (6a) is part of a longer discourse in which Fred and Mary are on an equal footing, for example (6b).

(6) a. Fred ordered salmon. Mary did too. Next, he had an apple pie. Structure $(X)$ with $R_{a}=$ Parallel and $R_{b}=$ Narration

b. Fred and Mary went to a fancy restaurant last night. Fred ordered salmon. Mary did too. Next, he had an apple pie. On the other hand, Mary had cheese cake.

In (6b), the narrative of Fred and Mary's dinner is organized following the dishes they ordered: first the main course, next the dessert. This narrative structure can be reflected in an SDRT graph in which the topic $\pi^{*}$ of the second and third sentences is Fred and Mary's main course to which is attached the topic $\pi^{* *}$ of the fourth and fifth sentences defined as Fred and Mary's dessert. This discourse structure is given in Figure 9. In this structure, the sub-discourse (6a) is not given structure (X).

In a nutshell, since (6a) illustrates the only pattern of felicitous discourses with structure (X) I can imagine, it would seem that this structure cannot be linguistically realized, provided that it is accepted that the coordinating/subordinating type of a discourse relation changes according to the context (for example, provided that it is accepted that the coordinating relation Parallel can be demoted down to a subordinating relation in context).

\footnotetext{
${ }^{12}$ In this structure, the third and fourth sentences form a complex constituent which elaborates/comments the complex constituent made up of the first and second sentences (which are linked by the relation Parallel/Narration).
} 


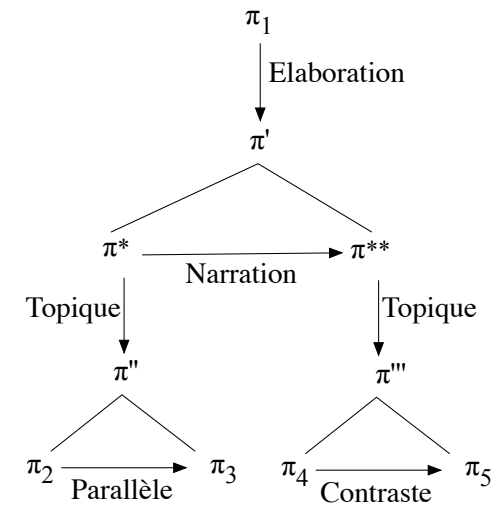

Figure 9: SDRT graph for the discourse in $(6 b)$

Linguistic realization of (XI) and (XII): discourses (7a) and (7b) illustrate respectively structures (XI) and (XII).

(7) a. Mary is worrying because her eldest son has bad marks. Her youngest son also has bad marks (but she doesn't care).

Structure $(X I)$ with $R_{a}=$ Explanation and $R_{b}=$ Parallel

b. Fred tidied up his bedroom this afternoon. Mary did too. Next she went to the movies.

Structure (XII) with $R_{a}=$ Parallel and $R_{b}=$ Narration

In SDRT, the discourse in (7a) cannot be given structure (XI): SDRT graph (XIc) is transformed into graph (VIIIc) in Table 2 by the Continuing Discourse Pattern Constraint (see above). For (7a), this constraint amounts to inferring that Mary is also worrying about the bad marks of her youngest son, although this may be wrong (as suggested by the element we put into brackets but she doesn't care). These data lead us to state that the Continuing Discourse Pattern Constraint could be maintained when the subordinating relation $R_{a}$ is Elaboration, but should not be maintained when $R_{a}$ is Explanation. This allows (7a) to be analyzed as (XIc).

We have seen that (XIc) and (XIIc) are not tree shaped (disregarding horizontal arrows) since the node $\pi_{2}$ has two parents. The felicitous examples in (7) show that non tree shaped SDRT graphs must be authorized.

\subsubsection{Tree shaped DAGs without any equivalent RST tree}

In the four tree shaped dependency DAGs in Table 2, namely (Vb)-(VIIIb), the embedded relation is coordinating. What can be said about the discourse structures corresponding to the four other tree shaped DAGs with an embedded subordinating relation? Consider DAG (XIIIb) in Figure 10 (this figure also includes the equivalent SDRT graph which is commented below). The Nucleus argument of $R_{b}$ in this DAG is the sub-tree 
rooted at $R_{a}$. This predicate-argument relation is not possible in (Marcu's version of) RST because of the Nuclearity Principle which states that the Nucleus argument of $R_{b}$ is $\pi_{1}$.

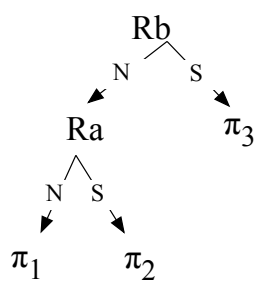

(XIIIb)

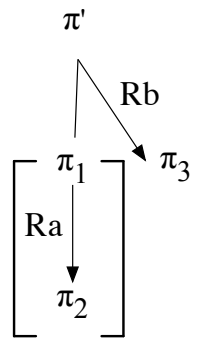

(XIIIc)

Figure 10: DAG (XIIIb) and its equivalent SDRT graph

The discourse structure (XIIIb) can be felicitously realized, for example in discourse (8), in which the antecedent of this is the interpretation of its left context, namely the causal relation linking the interpretations of the two first sentences.

(8) Fred is upset because his wife is abroad for a week. This proves that he does love her.

Structure $(X I I I b)$ with $R_{a}=$ Explanation and $R_{b}=$ Comment

The discourse structure for (8) is not controversial. In the framework of RST, Egg (pc.) indeed does analyze (8) as (XIIIb) and acknowledges that this is a clear counterexample to the systematic applicability of the Nuclearity Principle. In SDRT, Asher (pc.) analyzes (8) as (XIIIc), i.e. as a graph including brackets around $\pi_{1}, \pi_{2}$ and the arrow labelled $R_{a}$. These brackets mean that (XIIIc) must be interpreted as including a complex constituent formed by $\pi_{1}$ and $\pi_{2}$ linked by $R_{a}$. This is not yet formalized in the theory, in which only complex constituents formed around a coordinating discourse relation are formally handled in the present state of the formalism.

In summary, neither RST nor SDRT can accurately handle the structure of (8). This is explained by the fact that (8) does not fit with the basic principles of these theories which rely heavily on the coordinating/subordinating distinction and consider the relation Explanation as subordinating and the explanation of a fact as a satellite of lower importance. Yet, in (8), the explanation given for Fred's being upset is essential for the interpretation of the third sentence ${ }^{13}$.

The only formalism in which the structure of (8) does not raise any problem is the dependency DAG formalism, which goes along with the fact that this formalism does not make any use of the coordinating/subordinating distinction.

\footnotetext{
${ }^{13}$ Nevertheless, neither Egg nor Asher contemplate the solution consisting in promoting Explanation as a coordinating relation in an example such as (8).
} 
The three other tree shaped DAGs with an embedded subordinating relation raise the same questions as (XIIIb). So I will not comment on them in detail and I consider that they can all be linguistically realized in a felicitous way.

\section{Summary and Conclusion}

First, we give an assessment on the strong generative capacity of RST, SDRT and dependency DAG formalism, for discourses in the canonical order with three sentences.

There exist sixteen dependency DAGs which respect the minimal constraints $C_{1}$ and $C_{2}$ exposed in Section 4.

RST - in the version presented in Section 2 - authorizes only eight discourse structures, namely structures (I)-(VIII) in tables 1 and 2. These discourse structures can all be realized in felicitous discourses. However, RST is too restrictive: it excludes discourse structures which can be realized in felicitous discourses, as described below.

SDRT authorizes the eight discourse structures admitted by RST and the two structures (XI) ${ }^{14}$ and (XII), which are realized in felicitous discourses, cf. (7).

SDRT excludes structures (IX) and (X) because of the Right Frontier Constraint. The question of whether structure (IX) can be realized in felicitous discourses depends (at least) upon the status that should be given to anaphoric links: should they be systematically reflected in discourse structures or not (cf. the discussion of examples (4) and (5))? Concerning structure (X), it seems hard to find a felicitous discourse realizing it (see the discussion of (6)). If it turns out that this structure cannot indeed be realized in felicitous discourses, this is a strong argument in favor of the Right Frontier Constraint for the attachment of new information and, thereby, a strong argument against the dependency DAG formalism which cannot exclude structure (X).

We are left with structure (XIII) and the three other structures in which a subordinating relation forms a complex constituent. These structures can be realized in felicitous discourses, see (8). Only dependency DAG formalism authorizes them ${ }^{15}$.

In conclusion, none of the three formalisms under study - RST, SDRT, and dependency DAG formalisms - has the appropriate strong generative capacity. These formalisms are either too restrictive or too powerful. This conclusion may sound negative, however we hope this study will shed new light on the constraints which must hold on discourse structures and on the following open questions: should the distinction between the coordinating/subordinating type of discourse relations be kept? Should the type of a given discourse relation be fixed in a static way - which is easy to implement - or computed in a dynamic way according to the context - which is hard to implement and may give rise to vicious circles?

What can be expected about the strong generative capacity of these formalisms for discourses in the canonical order with more than three sentences? The same results as those obtained for discourses in the canonical order with three sentences. This is because constraints are too restrictive in RST, not restrictive enough in DAG formalism, and not totally adequate in SDRT, whatever the number of sentences.

\footnotetext{
${ }^{14}$ If the Continuous Discourse Pattern Constraint is applied in a limited way, which means that (XIc) is not excluded.

${ }^{15}$ SDRT is ready to authorize them, but does not yet have the formal mechanism to do it
} 
On the other hand, there is an unknown factor, namely the number of discourse structures which can be realized in felicitous discourses. Consider discourses in the canonical order with four sentences. There exist five non-labelled RST trees and twentyfive non-labelled dependency DAGs respecting constraints $C_{1}$ and $C_{2}$ for these discourses. This leads to forty labelled RST trees and two hundred labelled dependency DAGs. We cannot tell where the number of felicitous discourses stands between forty and two hundred.

Future research is needed for discourses which are not in canonical order, for example, for discourses with preposed subordinate clauses or discourses with multiple discourse connectives in the same clause.

Acknowledgments: I thank first Nicholas Asher (SDRT) and Markus Egg (RST) for their comments and the fruitful discussions we had in Malaga during ESSLI'2006. I also thank Sylvain Kahane for his invaluable help on Dependency structures. Finally, I thank André Bittar and Laure Vieu for their comments.

\section{References}

[Asher and Lascarides2003] Nicholas Asher and Alex Lascarides. 2003. Logics of Conversation. Cambridge University Press, Cambridge.

[Asher and Vieu2005] Nicholas Asher and Laure Vieu. 2005. Subordinating and coordinating discourse relations. Lingua, 115(4):591-610.

[Asher1993] Nicholas Asher. 1993. Reference to Abstract Objects in Discourse. Kluwer, Dordrecht.

[Asher2007] Nicholas Asher. 2007. Troubles on the right frontier. In A. Benz and P. Kühnlein, editors, Constraints in Discourse. Benjamins.

[Carlson et al.2003] Lynn Carlson, Daniel Marcu, and Mary Ellen Okurowski. 2003. Building a discourse-tagged corpus in the framework of rhetorical structure theory. In Jan van Kuppevelt and Ronnie Smith, editors, Current Directions in Discourse and Dialogue, pages 85-112. Kluwer Academic Publishers.

[Danlos2004] Laurence Danlos. 2004. Discourse dependency structures as constrained DAGs. In Proceedings of SIGDIAL'04, pages 127-135, Boston.

[Delort2006] Laurence Delort. 2006. Clause 'subordination' and discourse relations. In Proceedings of the 28th Annual Meeting of the German Society for Linguistics (DGfS-06), Workshop on Subordination vs. Coordination in Sentence and Text from a Cross-linguistic Perspective, Bielefeld, Germany.

[Egg and Redeker2007] Markus Egg and Gisela Redeker. 2007. Underspecified discourse representation. In A. Benz and P. Kühnlein, editors, Constraints in Discourse. Benjamins.

[Hobbs1979] Jerry Hobbs. 1979. Coherence and coreference. Cognitive Science, 6:67-90.

[Hunter et al.2006] Julie Hunter, Nicholas Asher, Brian Reese, and Pascal Denis. 2006. Evidentiality and intensionality: Two uses of reportive constructions in discourse. In Constraints in Discourse, pages 99-106, Maynooth, Ireland.

[Kahane2001] Sylvain Kahane. 2001. Grammaires de dépendance formelles et théorie senstexte. In Proceedings of Tutoriel of TALN, pages 17-76, Tours, France. 
[Kamp and Reyle1993] Hans Kamp and Uwe Reyle. 1993. From Discourse to Logic. Kluwer Academic Publishers, Dordrecht.

[Lecerf1961] Yves Lecerf. 1961. Une représentation algébrique de la structure des phrases dans diverses langues naturelles. Compte Rendu de l'Académie des Sciences, 252:232-34.

[Mann and Thompson1987] William Mann and Sandra Thompson. 1987. Rhetorical structure theory. In G. Kempen, editor, Natural Language Generation, pages 85-95. Martinus Nijhoff Publisher, Dordrecht.

[Mann and Thompson1988] William C. Mann and Sandra A. Thompson. 1988. Rhetorical structure theory: Toward a functional theory of text organization. Text, 8(3):243-281.

[Marcu1996] Daniel Marcu. 1996. Building up rhetorical structure trees. In The Proceedings of the 13th National Conference on Artificial Intelligence, pages 1069-1074, Portland.

[Marcu2000a] Daniel Marcu. 2000a. The rhetorical parsing of unrestricted texts: A surfacebased approach. Computational Linguistics, 26(3):395-448.

[Marcu2000b] Daniel Marcu. 2000b. The Theory and Practice of Discourse Parsing and Summarization. The MIT Press.

[Matthiessen and Thompson1988] Christian Matthiessen and Sandra Thompson. 1988. The structure of discourse and 'subordination'. In John Haiman and Sandra Thompson, editors, Clause Combining in Grammar and Discourse, volume 18 of Typological Studies in Language, pages 275-329. John Benjamins, Amsterdam/Philadelphia.

[Mel'cuk1988] Igor Mel'cuk. 1988. Dependency Syntax: Theory and Practice. State Univ. of NY Press, Albany.

[Polanyi1988] Livia Polanyi. 1988. A formal model of the structure of discourse. Journal of Pragmatics, 12:601-638.

[Prevot and Vieu2005] Laurent Prevot and Laure Vieu. 2005. The moving right frontier. In Proceedings of the Workshop on Constraints in Discourse, pages 136-142, Dortmund, Germany.

[Redeker and Egg2006] Gisela Redeker and Markus Egg. 2006. Says who? on the treatment of speech attributions in discourse structure. In Constraints in Discourse, pages 140-146, Maynooth, Ireland.

[Sag et al.1985] I. Sag, G. Gazdar, T. Wasow, and s. Wisler. 1985. Coordination and how to disinguish categories. Natural Language and Linguistic Theory, 3(2):117-171.

[Stede1999] Manfred Stede. 1999. Rhetorical structure and thematic structure in text generation. In Proceedings of LORID'99, pages 44-50.

[Stede2007] Manfred Stede. 2007. RST revisited: Disentangling nuclearity. In Subordination vs. Coordination in Sentence and Text from a Cross-linguistic Perspective.

[Taboada and Mann2006a] Maite Taboada and William Mann. 2006a. Applications of rhetorical structure theory. Discourse Studies, 8(4):567-588.

[Taboada and Mann2006b] Maite Taboada and William Mann. 2006b. Rhetorical structure theory: Looking back and moving ahead. Discourse Studies, 8(3):423-459.

[Webber et al.2001] Bonnie Webber, Alistair Knott, and Aravind Joshi. 2001. Multiple discourse connectives in a lexicalized grammar for discourse. In H. Bunt, R. Muskens, and E. Thijsse, editors, Computing Meaning, volume 2, pages 229-246. Kluwer Academic Press.

[Wolf and Gibson2005] Florian Wolf and Edward Gibson. 2005. Representing discourse coherence: a corpus-based study. Computational Linguistics, 31(3):249-287. 